\title{
LOCALLY FINITE AND LOCALLY NILPOTENT DERIVATIONS WITH APPLICATIONS TO POLYNOMIAL FLOWS AND POLYNOMIAL MORPHISMS
}

\author{
ARNO VAN DEN ESSEN
}

(Communicated by Maurice Auslander)

\begin{abstract}
We give a very simple proof of the fact that the Lorenz equations and the Maxwell-Bloch equations do not have a polynomial flow. We also give an algorithm to decide if a two-dimensional vector field over $\mathbb{R}$ has a polynomial flow and how to compute the solutions (in case the vector field has a polynomial flow).
\end{abstract}

\section{INTRODUCTION}

Locally nilpotent and locally finite derivations are hidden in many problems. However once their presence is revealed, information on these derivations can give valuable information on the problem under consideration. It is the aim of this paper to illustrate this statement with several examples.

The first examples we discuss in $\S 2$ come from the theory of polynomials flows (a solution of an autonomous system of ordinary differential equations $\dot{y}=V(y), y(0)=x \in \mathbb{R}^{n}$, where $V$ is a $C^{1}$ vector field on $\mathbb{R}^{n}$, is called a polynomial flow if the solution depends polynomially upon the initial condition). It was shown by Coomes in [3 and 4] that the Lorenz equations do not have a polynomial flow. In [5] Coomes and Zurkowski showed that the Maxwell-Bloch equations do not have a polynomial flow either. In their paper they prove the following fundamental result: let $V$ be a polynomial vector field and $D_{V}$ the corresponding derivation on the $n$ variable polynomial ring over $\mathbb{R}$. Then the autonomous system $\dot{y}=V(y), y(0)=x$ has a polynomial flow if and only if the derivation $D_{V}$ is locally finite. In this paper we give a very simple proof of the fact that the Lorenz system and the Maxwell-Bloch equations do not have a polynomial flow based on Coomes and Zurkowski's theorem and an easy but crucial property of locally finite derivations (which we call the second principle). Using this principle and results of Rentschler [12] we study locally finite derivations on polynomial rings in two variables over a field and obtain an algorithm that decides if a derivation is locally finite.

In $\S 5$, this result is used to give a complete solution of the recognition problem for polynomial flow vector fields in the plane; i.e., given a polynomial vector

Received by the editors April 8, 1991.

1991 Mathematics Subject Classification. Primary 58F25; Secondary 14E05. 
field $V$ in $\mathbb{R}^{2}$ we give an algorithm to decide if this vector field has a polynomial flow. Using this algorithm we obtain a new proof of the classification theorem of Bass and Meisters [2].

More precisely, given a polynomial flow vector field $V$ corresponding to the autonomous system $\dot{y}=V(y), y(0)=x \in \mathbb{R}^{2}$, a polynomial automorphism $\varphi$ of $\mathbb{R}[X, Y]$ is constructed such that $\varphi^{-1} D_{V} \varphi$ is one of the six vector fields described in the theorem of Bass and Meisters. Since the flows of these six types are well known, we can also calculate the solution of the system $\dot{y}=$ $V(y), y(0)=x$. So we obtain an algorithm to compute the solution of any autonomous system having a polynomial flow.

Section three is independent of the others. Its results are more or less known. First we describe how the automorphism group of a polynomial ring in two variables over a field can be obtained from a classification theorem of locally nilpotent derivations (these results are due to Rentschler [12]). Next we discuss the automorphism group of polynomial rings in more than two variables and describe a candidate generator set of "exotic" polynomial automorphisms. Finally, at the end of $\S 3$ we show how the Jacobian conjecture can be formulated in terms of locally nilpotent derivations.

\section{FundaMeNTAL PROPERTIES OF LOCALly FINITE AND LOCALLY NILPOTENT DERIVATIONS}

Throughout this paper we have the following notations: $k$ a field of characteristic zero, $R$ a commutative $k$-algebra, and $D$ a $k$-derivation of $R$.

We call $D$ locally finite if for every $g \in R$ the $k$-vector space generated by the elements $D^{i} g, i \in \mathbb{N}$, is finite dimensional. The derivation $D$ is called locally nilpotent if for every $g \in R$ there exists an integer $n \geq 1$ such that $D^{n} g=0$. From Leibniz's formula one obtains

Lemma 1.1. Let $G$ be a generating set for the $k$-algebra $R$. If for each $g \in$ $G$ the vector space generated by the elements $D^{i} g$ is finite dimensional (resp. $D^{n} g=0$ for some $n \geq 1$ ) then $D$ is locally finite (resp. locally nilpotent).

Example 1.2. Let $R=k[X]$, the one variable polynomial ring over $k$, and $0 \neq D$ a $k$-derivation of $R$, i.e., $D=a(X) \partial_{X}$ for some nonzero polynomial $a(X) \in R$. Then one readily verifies that $D$ is locally nilpotent if and only if $\operatorname{deg} a(X) \leq 0$ and $D$ is locally finite if and only if $\operatorname{deg} a(X) \leq 1$.

1.3. Two simple principles. Almost all results of this paper will be derived from the following two principles. To describe the first one (the proof of which is trivial) we denote by $R^{D}$ the set of constants of $D$, i.e., the set of elements $r$ in $R$ satisfying $D r=0$. Observe that $R^{D}$ is a $k$-sublagebra of $R$.

Principle I. Assume $R$ has no $R^{D}$-torsion. Let $S \subset R^{D}$ be a multiplicatively closed set. Then $D$ is locally nilpotent on $R$ if and only if $\widetilde{D}: S^{-1} R \rightarrow S^{-1} R$ is locally nilpotent (where $\widetilde{D}$ is the extended derivation defined by $\widetilde{D}\left(s^{-1} r\right)=$ $\left.s^{-1} \mathrm{Dr}\right)$.

1.4. An application. Let $R=k\left[X_{1}, \ldots, X_{n}\right]$ (the $n$ variable polynomial ring over $k$ ) and $f \in R$. Then $f \partial_{X_{1}}$ is locally nilpotent if and only if $f \in$ $k\left[X_{2}, \ldots, X_{n}\right]$ (take $S=k\left[X_{2}, \ldots, X_{n}\right] \backslash\{0\}$ and use Example 1.2). 
To describe the second principle we assume that $R=\bigoplus_{n \in \mathbb{Z}} R_{n}$ is a graded $k$-module. Hence each element $g \in R$ can be written uniquely as a sum of homogeneous elements $g=g_{r}+g_{r+1}+\cdots+g_{s}$, for some $g_{i} \in R_{i}$. If $g_{s} \neq 0$ we say that $g$ has degree $s$ and write $\operatorname{deg} g=s$. Suppose that $D$ can be written as a sum of derivations $D=D_{p}+D_{p+1}+\cdots+D_{d}$ (with $D_{d} \neq 0$ ) satisfying $D_{n} R_{m} \subset R_{n+m}$ for all $n, m$. Then we have

Principle II. If $D$ is locally finite of degree $d \neq 0$, then $D_{d}$ is locally nilpotent. Proof. It suffices to prove that each homogeneous element of $R$ is annihilated by a power of $D_{d}$. So let $n \in \mathbb{Z}$ and $g \in R_{n}$. Let $p \in \mathbb{N}$. If $D_{d}^{p} g \neq 0$ then

$$
\operatorname{deg} D^{p} g=\operatorname{deg} D_{d}^{p} g=\operatorname{deg} g+p d .
$$

Since $D$ is locally finite, the $k$-vector space generated by the elements $D^{p} g$, $p \geq 0$, has a finite number of generators, each of them being a finite sum of elements of some $R_{i}$. So there exists a positive integer $N$ such that all elements $D^{p} g$ are contained in $\bigoplus_{n=-N}^{N} R_{n}$. Then $(*)$ implies that if $p$ is such that $|\operatorname{deg} g+p d|>N$ then $D^{p} g=0$, which completes the proof.

\section{The LoRenz equations AND the MAXWell-Bloch EQUATions HAVE NO POLYNOMIAL FLOW}

As a first application of the results of $\S 1$ we show that the Lorenz equations and the Maxwell-Bloch equations have no polynomial flow.

First we recall some facts on polynomial flows (see $[9,2,5])$. Let $V$ be a $C^{1}$ vector field on $\mathbb{R}^{n}$, and consider the initial value problem

$$
\dot{y}=V(y), \quad y(0)=x \in \mathbb{R}^{n} .
$$

The solution (flow) $\varphi$ of (2.1) is said to be a polynomial flow if $\varphi$ depends polynomially upon the initial condition (i.e., $\varphi^{t}(x):=\varphi(t, x)$ is polynomial in $x$ for each $t$ where the solution is defined).

The vector field $V$ is then called a poly-flow vector field, abbreviated $\mathrm{p}-\mathrm{f}$ vector field. It is shown by Bass and Meisters in [2] that if $V$ is a p-f vector field, then the $C^{1}$ vector field $V$ itself is polynomial, the solutions are defined for all $t \in \mathbb{R}$, and $\varphi^{t}$ has a bounded degree in $x$, independent of $t$. Using these results, Coomes and Zurkowski give a purely algebraic description of a p-f vector field on $\mathbb{R}^{n}$ (or $\mathbb{C}^{n}$ ).

Let $V=\left(V_{1}, \ldots, V_{n}\right)$ be a polynomial vector field on $\mathbb{R}^{n}$, i.e., each $V_{i}$ belongs to the $n$-variable polynomial ring $\mathbb{R}[X]:=\mathbb{R}\left[X_{1}, \ldots, X_{n}\right]$. Let $D=$ $\sum V_{i} \partial_{X_{i}}$ be the derivation on $\mathbb{R}[X]$ associated to $V$.

Theorem 2.2 [5, Theorem 3.1]. $V$ is a p-f vector field if and only if $D$ is locally finite on $\mathbb{R}[X]$.

Now consider the Lorenz system

$$
\left\{\begin{array}{l}
\dot{x}=\sigma(y-x), \\
\dot{y}=\rho x-y-x z, \quad \sigma, \rho, \beta \in \mathbb{R} . \\
\dot{z}=-\beta z+x y,
\end{array}\right.
$$

Corollary 2.3. The Lorenz system has no polynomial flow.

Proof. Put $R=\mathbb{R}[X, Y, Z]$ and $D=\sigma(Y-X) \partial_{X}+(\rho X-Y-X Z) \partial_{Y}+$ $(-\beta Z+X Y) \partial_{Z}$. By Theorem 2.2 we need to show that $D$ is not locally finite. 
Therefore, let $R_{n}$ be the $\mathbb{R}$-vector space generated by the monomials of degree $n$. Then $R=\bigoplus R_{n}$ is a graded $\mathbb{R}$-module. Furthermore, write $D=D_{0}+D_{1}$ where $D_{0}=\sigma(Y-X) \partial_{X}+(\rho X-Y) \partial_{Y}+-\beta Z \partial_{Z}$ and $D_{1}=-X Z \partial_{Y}+X Y \partial_{Z}$. By Principle II it suffices to show that $D_{1}$ is not locally nilpotent, which, in turn, is equivalent to showing that $D_{1}^{\prime}$ is not locally nilpotent (where $D_{1}=X D_{1}^{\prime}$ ). But this is obvious since $D_{1}^{\prime}$ induces an $\mathbb{R}$-linear map on $\mathbb{R} Y+\mathbb{R} Z$ that is not nilpotent.

Now consider the Maxwell-Bloch equations

$$
\left\{\begin{array}{l}
\dot{x}=-y, \\
\dot{y}=x+\varepsilon z, \\
\dot{z}=-\varepsilon y, \\
\dot{\varepsilon}=\vartheta, \\
\dot{\vartheta}=-\varepsilon+\beta(x+\varepsilon z), \quad \beta \in \mathbb{R} .
\end{array}\right.
$$

Corollary 2.4. The Maxwell-Bloch equations have no polynomial flow.

Proof. Arguing as in Corollary 2.3, it remains to show that $D_{1}=\varepsilon Z \partial_{Y}-\varepsilon Y \partial_{Z}+$ $\beta \varepsilon Z \partial_{\vartheta}$ is not locally nilpotent on $\mathbb{R}[X, Y, Z, \varepsilon, \vartheta]$, which is equivalent to showing that $D_{1}^{\prime}=Z \partial_{Y}-Y \partial_{Z}+\beta Z \partial_{\vartheta}$ is not locally nilpotent. Therefore, observe that the restriction of $D_{1}^{\prime}$ to $\mathbb{R} Y+\mathbb{R} Z+\mathbb{R} \vartheta$ is a nonnilpotent linear map.

Comment. Corollary 2.3 was proved by Coomes in [4] and also by Coomes and Zurkowski in [5] under the restriction $\sigma \neq 0$. Corollary 2.4 was proved by Coomes and Zurkowski under the restriction $\beta \neq 0$.

\section{LOCALLY NILPOTENT DERIVATIONS AND POLYNOMIAL AUTOMORPHISMS}

In this section we give two examples of how locally nilpotent derivations play a role in the study of polynomial automorphisms. The first example concerns the structure of the automorphism group Aut $k[X]\left(k[X]:=k\left[X_{1}, \ldots, X_{n}\right]\right)$. The second example concerns the Jacobian Conjecture.

3.1. Locally nilpotent derivations and the structure of $\operatorname{Aut}_{k} k[X]$. Let $F$ be a polynomial morphism of $k[X]$. Then $F$ is called triangular if $F\left(X_{i}\right)-X_{i} \in$ $k\left[X_{i+1}, \ldots, X_{n}\right]$ for all $1 \leq i \leq n-1$ and $F\left(X_{n}\right)=X_{n}$. If, furthermore, $F\left(X_{i}\right)=X_{i}$ for all $1 \leq i \leq n-1$ with at most one exception then $F$ is called elementary. One readily verifies that a triangular $F$ is a finite product of elementary ones. Finally, a polynomial morphism that is a finite product of invertible linear polynomial morphisms and elementary ones is called tame.

Now let us consider the automorphism group $\mathrm{Aut}_{k} k[X]$ in more detail. If $n=1$ then Aut $_{k} k[X]$ consists of just the invertible linear polynomial morphisms. If $n=2$ then every element of $\mathrm{Aut}_{k} k[X]$ is tame. This result is due to Jung [7] and van der Kulk [8]. An elegant proof of this result was given by Rentschler in [12] using locally nilpotent derivations. He shows that every locally nilpotent derivation $D$ of $k\left[X_{1}, X_{2}\right]$ is conjugate, by a tame automorphism, to a derivation of the form $f\left(X_{2}\right) \partial_{X_{1}}$, where $f\left(X_{2}\right) \in k\left[X_{2}\right]$, i.e., there exists a tame polynomial automorphism $\varphi$ such that $\varphi^{-1} D \varphi=$ $f\left(X_{2}\right) \partial_{X_{1}}$ (see also $\S 4$ ). From this result the Jung-van der Kulk theorem follows readily: namely, let $F$ be a polynomial automorphism of $k[X]$. Let 
$F_{i}=F\left(X_{i}\right)$. so $k[X]=k\left[F_{1}, F_{2}\right]$ and $d / d F_{1}$ is locally nilpotent. So there exists a tame automorphism $\varphi$ and a polynomial $f\left(X_{2}\right) \in k\left[X_{2}\right]$ such that $\varphi^{-1} \circ \frac{d}{d F_{1}} \circ \varphi=f\left(X_{2}\right) \partial_{X_{1}}$. Computing their kernels gives $k\left[\varphi^{-1}\left(F_{2}\right)\right]=k\left[X_{2}\right]$, whence $F_{2}=\lambda_{1} \varphi\left(X_{2}\right)+\mu_{1}$ for some $\lambda_{1} \in k^{*}$ and $\mu_{1} \in k$. Furthermore, observe that $\varphi^{-1} \circ \frac{d}{d F_{1}} \circ \varphi\left(\varphi^{-1}\left(F_{1}\right)\right)=1$, whence $f\left(X_{2}\right) \partial_{X_{1}}\left(\varphi^{-1}\left(F_{1}\right)\right)=1$. So we get $\partial_{X_{1}}\left(\varphi^{-1}\left(F_{1}\right)\right) \in k^{*}$, implying $\varphi^{-1}\left(F_{1}\right)=\lambda_{2} X_{1}+\mu_{2}\left(X_{2}\right)$ with $\lambda_{2} \in k^{*}$ and $\mu_{2}\left(X_{2}\right) \in k\left[X_{2}\right]$, i.e., $F_{1}=\lambda_{2} \varphi\left(X_{1}\right)+\mu_{2}\left(\varphi\left(X_{2}\right)\right)$. From this formula and the formula $F_{2}=\lambda_{1} \varphi\left(X_{2}\right)+\mu_{1}$ above, one easily deduces that $F$ is tame.

If $n \geq 3$ then it seems likely that nontame automorphisms exist. For example, the following polynomial automorphism $\sigma$ of $k[X, Y, Z]$, constructed by Nagata, is a candidate for a nontame automorphism:

$$
\begin{aligned}
& \sigma(X)=X-2\left(X Z+Y^{2}\right) Y-\left(X Z+Y^{2}\right)^{2} Z, \\
& \sigma(Y)=Y+\left(X Z+Y^{2}\right) Z, \\
& \sigma(Z)=Z .
\end{aligned}
$$

In [1] Bass showed that $\sigma$ can be obtained using a locally nilpotent derivation of $k[X, Y, Z]$. His construction is easily generalized to $n$ variables (see also [13]) as follows.

Let $D$ be a locally nilpotent derivation of $k[X]$. Then we get a well-defined polynomial map of $k[X]$, denoted $\exp (D)$, by the formula

$$
\exp (D)(f)=\sum_{k=0}^{\infty} \frac{1}{k !} D^{k}(f), \quad \text { for all } f \in k[X]
$$

(the sum is finite since $D$ is locally nilpotent and the property $\exp (D)(f g)=$ $\exp (D)(f) \exp (D)(g)$ follows from Leibniz' rule). Since $\exp (D) \exp (-D)=$ Id it follows that $\exp (D)$ is a polynomial automorphism of $k[X]$.

To obtain Nagata's example, take $D=-2 Y \partial_{X}+Z \partial_{Y}$ and $a=X Z+Y^{2}$. Then $a \in k[X, Y, Z]^{D}$ and $D$ is locally nilpotent. It follows that $a D$ is locally nilpotent, and one easily verifies that $\sigma=\exp (a D)$. Also all elementary polynomial automorphisms of $k[X]$ can be obtained in a similar way; let $F$ be the elementary automorphism given by

$F\left(X_{i}\right)=X_{i}$ if $i \neq p$ and $F\left(X_{p}\right)=a\left(X_{p+1}, \ldots, X_{n}\right)$ for some $1 \leq p \leq n-1$.

Then $F=\exp \left(a \partial_{X_{p}}\right)$, as one readily verifies. Observe that $a \in k[X]^{\partial X_{p}}$.

Both derivations $D=-2 Y \partial_{X}+Z \partial_{Y}$ and $a\left(X_{p+1}, \ldots, X_{n}\right) \partial_{X_{p}}$ are examples of so-called triangular derivations; a derivation $D$ of $k[X]$ is called triangular if $D X_{i} \in k\left[X_{i+1}, \ldots, X_{n}\right]$ for all $1 \leq i \leq n-1$ and $D X_{n}=0$. A triangular derivation is locally nilpotent. This can be seen as follows: since $D X_{n}=0, D$ is locally nilpotent on $k\left[X_{n}\right]$ (Lemma 1.1). Since $D X_{n-1} \in k\left[X_{n}\right]$, it follows that $D^{p} X_{n-1}=0$ for some $p \in \mathbb{N}$. So $D$ is locally nilpotent on $k\left[X_{n}, X_{n-1}\right]$ (Lemma 1.1). Continuing in this way, we obtain that $D$ is locally nilpotent on $k[X]$.

These observations lead us to

Definition 3.2. A polynomial automorphism $F$ of $k[X]$ is called semi-tame if it is a finite product of invertible linear polynomial automorphisms and polynomial automorphisms of the form $\exp (a D)$ where $a \in k[X]^{D}$ and $D$ is a triangular derivation of $k[X]$. 
Problem. Is every polynomial automorphism semi-tame?

As observed before it is not known if the Nagata example is tame. However it was shown by M. Smith that it is stably tame; a polynomial $F$ of $k[X]$ is called stably tame if for some choice of coordinates $X_{n+1}, \ldots, X_{m}$ the extension of $F$ to $k\left[X_{1}, \ldots, X_{m}\right]$, which fixes $X_{n+1}, \ldots, X_{m}$, is tame.

Theorem 3.3 [13]. A semi-tame polynomial automorphism is stably tame. More precisely, let $D$ be a triangular derivation of $k[X]$. Extend $D$ to $k[X][t]$ by setting $D t=0$. If $a \in k[X]^{D}$ then

$$
\exp (a D)=\tau^{-1} \exp (-t D) \tau \exp (t D)
$$

where $\tau \in$ Aut $_{k} k[X][t]$ is defined by $\tau X_{i}=X_{i}$ for all $i$ and $\tau t=t+a$.

3.4. Locally nilpotent derivations and the Jacobian conjecture. Let $F$ be a polynomial morphism of $k[X], F_{i}=F\left(X_{i}\right)$. Let $J F=\left(\partial F_{j} / \partial X_{i}\right)$ be the Jacobian matrix of $F$. It is not difficult to verify that if $F$ is a polynomial automorphism then $\operatorname{det} J F \in k^{*}$. Conversely, we have the

Jacobian Conjecture. If $\operatorname{det} J F \in k^{*}$ then $F$ is a polynomial automorphism.

We conclude this section with a (well-known) equivalent formulation of this conjecture in terms of locally nilpotent derivations (see Corollary 3.7). Therefore, first observe that if $F$ is a polynomial automorphism, then each $d / d F_{i}$ is a locally nilpotent derivation of $k[X]$. Conversely, suppose $F$ is a polynomial morphism with det $J F \in k^{*}$. Then we define derivations $d_{1}, \ldots, d_{n}$ of $k[X]$ by the formula (see [11])

$$
\left(\begin{array}{c}
d_{1} \\
\vdots \\
d_{n}
\end{array}\right)=\left((J F)^{-1}\right)^{T}\left(\begin{array}{c}
\partial / \partial X_{1} \\
\vdots \\
\partial / \partial X_{n}
\end{array}\right) .
$$

Observe that $d_{i}\left(F_{j}\right)=\delta_{i j}$, for all $1 \leq i, j \leq n$.

Lemma 3.6. If each $d_{i}$ is locally nilpotent on $k[X]$ then $k[X]=k[F]$.

Proof. We may assume $F_{i}(0)=0$ for all $i$, and hence $k[[X]]=k[[F]]$ by the local (formal) inverse function theorem. The derivations $d_{i}$ can be extended uniquely to the formal power series ring $k[[F]]$. Let $g \in k[X]$ and $1 \leq i \leq n$. Then $d_{i}^{p} g=0$ for some $p \in \mathbb{N}$. Viewing $g$ as a formal power series in $k[[F]]$, it follows that $g$ is a polynomial in $F_{i}$ with degree $\leq p-1$. This holds for all $i$. So $g \in k[F]$, implying $k[X]=k[F]$.

Since $d_{i}=d / d F_{i}$ in case $F$ is a polynomial automorphism of $k[X]$ (both derivations agree on $k[F]=k[X]$ !), we obtain

Corollary 3.7. Let $F$ be a polynomial morphism with $\operatorname{det} J F \in k^{*}$. Then $F$ is a polynomial automorphism if and only if all $d_{i}$ are locally nilpotent. Consequently the Jacobian Conjecture is equivalent with: $\operatorname{det} J F \in k^{*}$ implies all $d_{i}$ are locally nilpotent.

Remark 3.8. It is shown in [11] that $d_{1}, \ldots, d_{n}$ are locally nilpotent on $k[X]$ if and only if they are locally finite on $k[X]$. In fact using results from the theory of $\mathscr{D}$-modules as described in [6], it can be shown that this last condition is even equivalent with the following condition: for each $1 \leq i \leq n$ and each prime 
ideal $p$ of height one in $k[F]$, any element $g \in k[X]$ satisfies an equation of the form $d_{i}^{r} g+a_{r-1} d_{i}^{r-1} g+\cdots+a_{0} g=0$ for some $r \in \mathbb{N}$ and $a_{j} \in k[F]_{p}$.

\section{AN ALGORITHM TO DECIDE IF A DERIVATION ON $k[X, Y]$ IS LOCALLY FINITE}

In this section we consider the polynomial ring $R=k[X, Y]$. To each $\varphi=(a, b) \in \mathbb{Z}^{2}$ we define a $\mathbb{Z}$-graduation on $R$, also called the $\varphi$-graduation by putting $R_{n}=\bigoplus_{a i+b j=n} k X^{i} Y^{j}$. So all points $(i, j)$ on the line $a x+b y=n$ are homogeneous of degree $n$. Let $D$ be a $k$-derivation of $R$. Then we can write $D=\sum_{\sigma, \tau \in \mathbb{Z}} D_{\sigma, \tau}$ (finite sum) where $D_{\sigma, \tau}$ is a derivation satisfying $D_{\sigma, \tau} k X^{i} Y^{j} \subset k X^{i+\sigma} Y^{j+\tau}$ for all $i, j$. Furthermore, corresponding to the $\varphi$ graduation on $R$, we put $D_{\rho}=\sum_{a \sigma+b \tau=\rho} D_{\sigma, \tau}$. Then we have $D=\sum_{\rho \in \mathbb{Z}} D_{\rho}$ (finite sum) and $D_{\rho} R_{n} \subset R_{n+\rho}$ for all $n, \rho \in \mathbb{Z}$. If $\rho$ is maximal with $D_{\rho} \neq 0$ then $\rho$ is called the $\varphi$-degree of $D$. In case $\varphi=(1,1) \rho$ is called the degree of $D$, denoted $\operatorname{deg} D$. Put

$$
\operatorname{supp} D=\left\{(\sigma, \tau) \mid D_{\sigma, \tau} \neq 0\right\} .
$$

So $\sigma, \tau \geq-1$ for all $(\sigma, \tau) \in \operatorname{supp} D$, and, for example, $(\sigma,-1) \in \operatorname{supp} D$ means that the term $X^{\sigma} \partial_{Y}$ appears in $D$ with nonzero coefficient.

Proposition 4.1 [12]. If $D=a \partial_{X}+b \partial_{Y}$ is locally nilpotent with $a, b \in R$ and $a b \neq 0$, then $\operatorname{supp} D$ contains points of the form $(\sigma,-1)$ and $(-1, \tau)$, with $\sigma, \tau \geq 0$.

Corollary 4.2. If $D$ is locally finite of degree $\rho_{0} \geq 1$, then the line $x+y=\rho_{0}$ contains a point of $\operatorname{supp} D$ of the form $(\sigma,-1)$ or of the form $(-1, \tau)$.

Proof. By principle II $D_{\rho_{0}}$ is locally nilpotent. If $a b \neq 0$ then apply Proposition 4.1. If $a=0$ then by 1.4 we get $D \rho_{0}=b(X) \partial_{Y}$, in which case the result easily follows. The case $b=0$ is treated similarly.

In case $\operatorname{supp} D$ contains a point of the form $(\sigma,-1)$ and a point $(-1, \tau)$ we define

$\sigma_{0}(D)=\max \{\sigma \mid(\sigma,-1) \in \operatorname{supp} D\}, \quad \tau_{0}(D)=\max \{\tau \mid(-1, \tau) \in \operatorname{supp} D\}$.

Proposition 4.3. Let $D$ be locally finite of degree $\rho_{0} \geq 1$, and suppose $\left(\sigma_{0},-1\right)$ $\in \operatorname{supp} D \cap\left\{x+y=\rho_{0}\right\} \quad$ (so $\sigma_{0} \geq 2$ ). Then either

(I) $\operatorname{supp} D$ contains no point of the form $(-1, \tau)$ so $D=\alpha X \partial_{X}+\beta Y \partial_{Y}+$ $f(X) \partial_{Y}$ with $\alpha, \beta \in k$ and $\operatorname{deg} f(X)=\sigma_{0} ;$ or

(II) $\operatorname{supp} D$ contains a point of the form $(-1, \tau)$ and $\tau_{0}(D)=0$ so $D=$ $\lambda \partial_{X}+\alpha X \partial_{X}+\beta Y \partial_{Y}+f(X) \partial_{Y}$ with $\lambda \in k^{*}, \alpha, \beta \in k$ and $\operatorname{deg} f(X)=$ $\sigma_{0} ;$ or

(III) $\operatorname{supp} D$ contains a point of the form $(-1, \tau)$ and $\tau_{0}(D)>0$ so $\operatorname{supp} D$ $\subset \operatorname{Tr} D:=$ the triangle with vertices $\left(\sigma_{0}(D),-1\right),(-1,-1),\left(-1, \tau_{0}(D)\right)$.

Proof. (i) Let $m$ be the line $x+y=\rho_{0}$. Suppose we are in case (I). Turn the line $m$ counterclockwise around $\left(\sigma_{0},-1\right)$ until you meet a point of $\operatorname{supp} D$ $\left(\neq\left(\sigma_{0},-1\right)\right)$. If you do not meet any point of $\operatorname{supp} D, \operatorname{supp} D=\left\{\left(\sigma_{0},-1\right)\right\}$, implying that $D=\lambda X^{\sigma_{0}} \partial_{Y}$ for some $\lambda \in k^{*}$. If you only meet points of $\operatorname{supp} D$ of the form $\left(\sigma^{\prime},-1\right)$, then $\sigma^{\prime} \leq \sigma_{0}$ and $D=f(X) \partial_{Y}$ with $\operatorname{deg} f(X)=\sigma_{0}$. So we may assume that we meet a point $\left(\sigma^{\prime}, \tau^{\prime}\right) \in \operatorname{supp} D$ with $\tau^{\prime} \geq 0$. By the 
hypothesis we know $\sigma^{\prime} \neq-1$, so $\sigma^{\prime} \geq 0$. There remain two cases. Case 1 . ( 0 , 0 ) is the only point of $\operatorname{supp} D$ you meet with $\left(\sigma^{\prime}, \tau^{\prime}\right) \geq 0$. Then $\operatorname{supp} D$ is contained in the triangle with vertices $(0,0),(0,-1),\left(\sigma_{0},-1\right)$ and $D$ is as described in (I). Case 2. Suppose now that $\sigma^{\prime}, \tau^{\prime} \geq 0$ with not both elements zero. By definition of $\left(\sigma^{\prime}, \tau^{\prime}\right)$ all points of $\operatorname{supp} D$ are below or on the line through $\left(\sigma_{0},-1\right)$ and $\left(\sigma^{\prime}, \tau^{\prime}\right)$. Then by Lemma 4.5 this line contains a point of supp $D$ of the form $(1, \tau)$, a contradiction with the hypothesis, which proves case (I).

(ii) Now assume that $\operatorname{supp} D$ also contains a point of the form $(-1, \tau)$. Let $\tau_{0}=\tau_{0}(D)$ and observe that $\sigma_{0}=\sigma_{0}(D)$. Let $l$ be the line through $\left(\sigma_{0},-1\right)$ and $\left(-1, \tau_{0}\right)$, i.e., $l:\left(\tau_{0}+1\right) x+\left(\sigma_{0}+1\right) y=\tau_{0} \sigma_{0}-1$. We will show

$$
\text { If }\left(\sigma^{\prime}, \tau^{\prime}\right) \in \operatorname{supp} D \text { lays above } l \text { then }\left(\sigma^{\prime}, \tau^{\prime}\right)=(0,0) \text {. }
$$

Let us assume (4.4) and show how we can finish the proof: therefore observe that if $(0,0)$ lays above $l$, then $\left(\tau_{0}+1\right) 0+\left(\sigma_{0}+1\right) 0>\tau_{0} \sigma_{0}-1$. Since $\sigma_{0} \geq 2$, this implies that $\tau_{0}=0$. So in case (III) there are no points of $\operatorname{supp} D$ laying above $l$ by (4.4), i.e., $\operatorname{supp} D \subset \operatorname{Tr} D$. Finally if $\tau_{0}=0$ the only possible point of $\operatorname{supp} D$ laying above $l$ is $(0,0)$ by $(4.4)$, whence supp $D \subset \operatorname{Tr} D \cup\{(0,0)\}$, which implies the statement of case (II) since $\operatorname{Tr} D=$ the triangle with vertices $\left(\sigma_{0},-1\right),(-1,-1),(-1,0) \quad\left(\tau_{0}=0 !\right)$.

Finally we prove (4.4): let $\left(\sigma^{\prime}, \tau^{\prime}\right) \in \operatorname{supp} D$ lay above $l$. Then $\sigma^{\prime} \geq 0$ (because of the choice of $\left.\tau_{0}\right)$. Similarly $\tau^{\prime} \geq 0$. Suppose now that $\left(\sigma^{\prime}, \tau^{\prime}\right) \neq$ $(0,0)$. Then turn the line through $\left(\sigma^{\prime}, \tau^{\prime}\right)$ and $\left(\sigma_{0},-1\right)$ clockwise around the point $\left(\sigma_{0},-1\right)$ in the direction of the line $m\left(x+y=\rho_{0}=\sigma_{0}-1\right)$ and stop when this line meets for the last time a point of $\operatorname{supp} D\left(\neq\left(\sigma_{0},-1\right)\right)$. Call this line $q$ (there may be more than one point of $\operatorname{supp} D$ on $q$ ). The line $q$ can be written as $\alpha x+\beta y=\rho$ for some $\alpha, \beta>0, \alpha, \beta \in \mathbb{Z}$ and contains a point $\left(\sigma^{\prime \prime}, \tau^{\prime \prime}\right)$ with $\sigma^{\prime \prime}, \tau^{\prime \prime} \geq 0$ and not both zero. Then by Lemma 4.5 the line $q$ contains a point of the form $(-1, \tau)$ of $\operatorname{supp} D$. By construction the line $q$ lays above $l$, whence $\tau>\tau_{0}$ a contradiction with the choice of $\tau_{0}$. This concludes the proof of (4.4) and hence of Proposition 4.3.

Lemma 4.5. Let $l$ be the line $\alpha x+\beta x=\rho$ with $\alpha, \beta>0, \alpha, \beta \in \mathbb{N}$, and $\rho \in \mathbb{R}$. Let $D$ be a locally finite derivation on $R$. Suppose that all points of supp $D$ are under or on the line $l$ and that $l$ contains a point $\left(\sigma_{0},-1\right)$ of supp $D$ with $\sigma_{0} \geq 2$ and a point $\left(\sigma^{\prime}, \tau^{\prime}\right)$ of $\operatorname{supp} D$ with $\sigma^{\prime}, \tau^{\prime} \geq 0$ and not both zero. Then $l$ contains a point of $\operatorname{supp} D$ of the form $(-1, \tau)$.

Proof. Since $\left(\sigma^{\prime}, \tau\right) \in l$ we have $\rho>0$. Now consider on $R$ the $(\alpha, \beta)$ graduation. Since all points of $\operatorname{supp} D$ are under or on the line $l, D_{\rho}(=$ the principal part of $D$ with respect to the $(\alpha, \beta)$-graduation) is locally nilpotent by Principle II. Since $\left(\sigma_{0},-1\right) \in l, D_{\rho}$ contains a term of the form $\lambda X^{\sigma_{0}} \partial_{Y}$ with $\lambda \in k^{*}$. However $D_{\rho}$ is not of the form $b(X, Y) \partial_{Y}$ (for otherwise $D_{\rho}=$ $b(X) \partial_{Y}$ by 1.4 and hence the only points of $\operatorname{supp} D_{\rho}$ are of the form $(\sigma,-1)$. However $\left(\sigma^{\prime}, \tau^{\prime}\right) \in l \cap \operatorname{supp} D=\operatorname{supp} D_{\rho}$ and $\tau^{\prime} \geq 0$, a contradiction). So $D_{\rho}=a \partial_{X}+b \partial_{Y}$ with $a b \neq 0$. Now apply Proposition 4.1.

Corollary 4.6. If $D$ is locally finite, then there exists an automorphism $\gamma$ of $R$ such that $\gamma D \gamma^{-1}$ is of the form $\lambda \partial_{X}+\alpha X \partial_{X}+\beta Y \partial_{Y}+f(X) \partial_{Y}$ with $\lambda, \alpha, \beta \in k$ and $f(X) \in k[X]$, or $\operatorname{deg} D \leq 0$. 
Proof. Suppose $\rho_{0}=\operatorname{deg} D \geq 1$. By Corollary 4.2 and if necessary a coordinate change sending $X$ to $Y$ and $Y$ to $X$, we may assume that we are in the situation of Proposition 4.3. We only need to consider case (III). So all points of $\operatorname{supp} D$ are on or below the line $l:\left(\tau_{0}+1\right) x+\left(\sigma_{0}+1\right) y=\sigma_{0} \tau_{0}-1 \quad\left(\tau_{0}:=\tau_{0}(D)\right)$. Put $\rho_{0}:=\sigma_{0} \tau_{0}-1$ and $\varphi=\left(\tau_{0}+1, \sigma_{0}+1\right)$, and consider the $\varphi$-graduation on $R$. Then by Principle II, $D_{\rho_{0}}$ is locally nilpotent. So by $\left[12,5^{\circ}\right]$ we get $D_{\rho_{0}}=g D_{1}$ with $D_{1} g=0$ and the ideal $\left(D_{1} R\right)$ equals $R$. Since $D_{\rho_{0}}$ is $\varphi$ homogeneous, so are $g$ and $D_{1}$. Since $\left(D_{1} R\right)=R, D_{1}$ contains either a term of the form $\lambda \partial_{X} \lambda \in k^{*}$, or a term $\mu \partial_{Y}, \mu \in k^{*}$. Let us assume we have the first case. Then $D_{1}=\lambda \partial_{X}+\mu X^{r} \partial_{Y}$ since $\left(\sigma_{0},-1\right) \in \operatorname{supp} D$ and $D_{1}$ is $\varphi$-homogeneous. Consequently $g \in k\left[Y-\mu X^{r+1} /(r+1) \lambda\right]$ (since $\left.D_{1} g=0\right)$. From $g \varphi$-homogeneous it follows that $g=\nu\left(Y-\mu X^{r+1} /(r+1) \lambda\right)^{p}$ for some $\nu \in k^{*}$ and $p \in \mathbb{N}$. So $D_{\rho_{0}}=\nu\left(Y-\mu X^{r+1} /(r+1) \lambda\right)^{p}\left(\lambda \partial_{X}+\mu X^{r} \partial_{Y}\right)$. Finally let $\gamma$ be the polynomial automorphism defined by

$$
\gamma(X)=X, \quad \gamma(Y)=Y+\mu X^{r+1} /(r+1) \lambda .
$$

Then $\gamma D_{\rho_{0}} \gamma^{-1}=\nu \lambda Y^{p} \partial_{X}$. Since $\gamma$ respects the $\varphi$-graduation on $R$, it follows that $\gamma D \gamma^{-1}=\sum_{\rho \leq \rho_{0}} \gamma D_{\rho} \gamma^{-1}$ is the $\varphi$-decomposition of $\gamma D \gamma^{-1}$. If $\operatorname{deg} \gamma D \gamma^{-1} \leq 0$ then we are done. If not then we may assume (as indicated above) that we are in the situation of Proposition 4.3(III). However $\sigma_{0}\left(\gamma D \gamma^{-1}\right)$ $<\sigma_{0}(D)$ since $\gamma D_{\rho_{0}} \gamma^{-1}=\nu Y^{p} \partial_{X}$ and the $\varphi$-decomposition of $\gamma D \gamma^{-1}$ given above. So by induction on $\sigma_{0}(D)+\tau_{0}(D)$, we can finish the proof.

Corollary 4.7. If $D$ is locally finite, then there exists an automorphism $\varphi$ of $R$ such that $\varphi D \varphi^{-1}$ is one of the following forms:

(i) $D=\left(\alpha X+\alpha^{\prime} Y\right) \partial_{X}+\left(\beta X+\beta^{\prime} Y\right) \partial_{Y}, \alpha, \alpha^{\prime}, \beta, \beta^{\prime} \in k$;

(ii) $D=\partial_{X}+\beta Y \partial_{Y}$;

(iii) $D=\alpha X \partial_{X}+\left(\alpha m Y+X^{m}\right) \partial_{Y}, m=1,2, \ldots$;

(iv) $D=f(X) \partial_{Y}$.

Proof. The case $\operatorname{deg} D \leq 0$ is readily reduced to cases (i) and (ii). So by Corollary 4.6 we may assume that $D$ is of the form $\lambda \partial_{X}+\alpha X \partial_{X}+\beta Y \partial_{Y}+$ $f(X) \partial_{Y}$

Step 1. $\quad \alpha \neq 0$. Let $\gamma(X)=X-\lambda / \alpha, \quad \gamma(Y)=Y$. Then $D^{\prime}:=$ $\gamma D \gamma^{-1}=\alpha X \partial_{X}+\beta Y \partial_{Y}+g(X) \partial_{Y}$ (where $\left.g(X)=f(X-\lambda / \alpha)\right)$. If $g=0$ we are in case (i). So let $g \neq 0$. Let $\gamma(X)=X, \gamma(Y)=Y+\sum_{m \neq \alpha^{-1} \beta}-$ $(\beta-m \alpha)^{-1} g_{m} X^{m}$, where $g=\sum g_{m} X^{m}$. Then $D^{\prime \prime}:=\gamma D^{\prime} \gamma^{-1}=\alpha X \partial_{X}+$ $\left(\alpha_{m} Y+g_{m} X^{m}\right) \partial_{Y}$. If $g_{m}=0$ we are in case (i). If $g_{m} \neq 0$ let $\gamma(X)=X$, $\gamma(Y)=g_{m} Y$. Then $\gamma^{-1} D^{\prime \prime} \gamma=\alpha X \partial_{X}+\left(\alpha m Y+X^{m}\right) \partial_{Y}$. If $m=0$ change $X$ and $Y$ and we are in case (ii). If $m>0$ we are in case (iii).

Step 2. $\alpha=0$. First assume $\lambda=0$. If $\beta=0$ we are in case (iv). If $\beta \neq 0$, let $\gamma(X)=X, \gamma(Y)=Y-\beta^{-1} f(X)$. Then $\gamma D \gamma^{-1}=\beta Y \partial_{Y}$, so we are in case (i). Assume $\lambda \neq 0$. Let $(d / d X) F(X)=f(X)$. If $\beta=0$, let $\gamma(X)=X$, $\gamma(Y)=Y+\lambda^{-1} F(X)$. Then $D^{\prime}:=\gamma D \gamma^{-1}=\lambda \partial_{X}$. Let $\gamma(X)=\lambda X, \gamma(Y)=Y$. Then $\gamma D^{\prime} \gamma^{-1}=\partial_{X}$, so we are in case (ii). Finally assume $\beta \neq 0$. Let $\gamma(X)=$ $X, \gamma(Y)=Y-\beta^{-1} f(X)$. Then $\gamma D \gamma^{-1}=\lambda \partial_{X}+\left(\beta Y+\beta^{-1} f^{\prime}(X)\right) \partial_{Y}$. So $\gamma D \gamma^{-1}$ is of the same form as $D$, however, $\operatorname{deg} \beta^{-1} f^{\prime}(X)<\operatorname{deg} f(X)$. So by induction on $\operatorname{deg} f$, we finally arrive at the form of case (ii), which completes the proof. 
Corollary 4.8. If $k=\mathbb{R}$ we obtain the six normal forms described by the classification theorem of Bass and Meisters [2].

Proof. One only needs to consider case (i) of Corollary 4.7 and use the Jordan form of a linear map. The details are left to the reader.

\section{A SOLUTION TO THE RECOGNITION PROBLEM IN DIMENSION TWO AND AN ALGORITHM TO SOLVE TWO-DIMENSIONAL AUTONOMOUS SYSTEMS HAVING A POLYNOMIAL FLOW}

Recognition Problem [10, Question 9]. Given a polynomial vector field $V$ on $\mathbb{R}^{n}$, how can we decide whether or not its flow $\varphi(t, x)$ is a polynomial flow?

Solution in dimension two. By Theorem 2.2 it suffices to give an algorithm to decide if $D_{V}$ is locally finite. From Corollary 4.2, Proposition 4.3, and Corollary 4.6, we obtain

5.1. Algorithm to decide if a derivation on $k[X, Y]$ is locally finite. Step 1. If $\operatorname{deg} D \leq 0, D$ is locally finite.

Step 2. Put $W_{1}=\operatorname{supp} D \cap\{\sigma=-1\}, W_{2}=\operatorname{supp} D \cap\{\tau=-1\}, W=$ $W_{1} \cup W_{2}$. If $W=\varnothing, D$ is not locally finite. If $W_{1} \neq \varnothing$ and $W_{2} \neq \varnothing$, compute $\sigma_{0}(D)$ and $\tau_{0}(D)$. Changing $X$ and $Y$ if necessary, we may assume $\sigma_{0} \geq \tau_{0}$. If $\tau_{0}=0$, check if $D$ is of the form $D=\lambda \partial_{X}+\alpha X \partial_{X}+\beta Y \partial_{Y}+f(X) \partial_{Y}$. If yes, $D$ is locally finite; if not, $D$ is not locally finite. If $\tau_{0}>0$, check if $\operatorname{supp} D \subset \operatorname{Tr} D$. If not, $D$ is not locally finite. If yes, compute $\rho_{0}=\sigma_{0} \tau_{0}-1$ and $D_{\rho_{0}}\left(=\sum_{(\sigma, \tau) \in l} D_{\sigma, \tau}\right)$ where $l$ is the line $\left(\tau_{0}+1\right) x+\left(\sigma_{0}+1\right) y=\rho_{0}$.

Write $D_{\rho_{0}}=a \partial_{X}+b \partial_{Y}$ and compute $g=\operatorname{gcd}(a, b)$ and write $D_{\rho_{0}}=g D_{1}$. Then, replacing $X$ by $Y$ if necessary we find that $D_{1}=\lambda \partial_{X}+\mu X^{\nu} \partial_{Y}$ with $\lambda \in k^{*}$. Then replace $D$ by $\gamma D \gamma^{-1}$ where $\gamma(X)=X$ and $\gamma(Y)=Y+$ $\mu X^{\nu+1} /(\nu+1) \lambda$ and return to Step 1 .

Step 3. If $W_{2} \neq \varnothing$ and $W_{1}=\varnothing$ compute $\sigma_{0}(D)$ and check if $\operatorname{supp} D \subset$ $\operatorname{Tr}\left((0,0),(-1,-1),\left(\sigma_{0},-1\right)\right)$. If yes, $D$ is locally finite; if not, $D$ is not locally finite.

Step 4. If $W_{1} \neq \varnothing$ and $W_{2}=\varnothing$ change $X$ and $Y$ and return to Step 3.

Corollary 5.2. Let $\dot{y}=V(y), y(0)=x \in \mathbb{R}^{2}$ be an autonomous system having a polynomial flow. Then its flow can be computed by the algorithms given in 5.1 and Corollary 4.7.

Namely, let $D_{V}$ be the derivation on $\mathbb{R}[X, Y]$ corresponding to the vector field $V$. By Theorem 2.2 $D_{V}$ is locally finite. So by 5.1 and Corollary 4.7 we can construct an automorphism $\gamma$ of $\mathbb{R}[X, Y]$ such that $\gamma D_{V} \gamma^{-1}$ is one of the forms described in Corollary 4.7. In each of these cases the flow $\varphi(t, x)$ of $\gamma^{-1} D_{V} \gamma$ can be computed explicitly (see [2]). Then the flow $\psi(t, x)$ of the original system is given by the formula $\psi(t, x)=\gamma^{-1}(\varphi(t, \gamma(x)))$.

\section{ACKNOWLEDGMENT}

I thank Ron Sommeling (University of Nijmegen) who kindly programmed the algorithm 5.1 in the computer algebra system Maple. Finally, I thank both my wife Sandra and our daughter Raïssa for creating a warm home in which living is a privilege. 


\section{REFERENCES}

1. H. Bass, A non-triangular action of $G_{a}$ on $\mathbb{A}^{3}$, J. Pure Appl. Algebra 33 (1984), 1-5.

2. H. Bass and G. H. Meisters, Polynomial flows in the plane, Advances in Math. 55 (1985), 173-208.

3. B. Coomes, Polynomials flows, symmetry groups, and conditions sufficient for injectivity of maps, PhD thesis, Univ. of Nebraska-Lincoln, 1988.

4. _ The Lorenz system does not have a polynomial flow, J. Differential Equations 82 (1989), 386-407.

5. B. Coomes and V. Zurkowski, Linearization of polynomial flows and spectra of derivations, J. Dynamics Differential Equations 3 (1990), 29-66.

6. A. van den Essen, $\mathscr{D}$-modules and the Jacobian Conjecture, Report 9108, Catholic Univ. Nijmegen; Proc. Internat. Conf. $\mathscr{D}$-Modules and Microlocal Geometry, Lisbon, Portugal, October 1990 (to appear).

7. H. Jung, Über ganze birationale Transformationen der Ebene, J. Reine Angew. Math. 184 (1942), 161-174.

8. W. van der Kulk, On polynomial rings in two variables, Nieuw Archief voor Wiskunde (3) I (1953), 33-41.

9. G. Meisters, Jacobian problems in differential equations and algebraic geometry, Rocky Mountain J. Math. 12 (1982), 679-705.

10. __ Polynomial flows on $\mathbb{R}^{n}$, Banach center publications, Vol. 23, Warzawa, 1989.

11. P. Nousiainen and M. Sweedler, Automorphisms of polynomial and power series rings, J. Pure Appl. Algebra 29 (1983), 93-97.

12. R. Rentschler, Opérations du groupe additif sur le plane affine, C. R. Acad. Sci. Paris $\mathbf{2 6 7}$ (1968), 384-387.

13. M. Smith, Stably tame automorphisms, J. Pure Appl. Algebra 58 (1989), 209-212.

Department of Mathematics, Catholic University, Toernooiveld, 6525 ED Nijmegen, The Netherlands

E-mail address: essen@sci.kun.nl 